\title{
HIV-I subtype C viruses rapidly develop K65R
}

\section{Mark A Wainberg*1, Florence Doualla-Bell ${ }^{1}$, Tedani Gaolatte ${ }^{1}$, Madisa Mine ${ }^{2}$, Max Essex ${ }^{2}$ and Bluma Brenner ${ }^{1}$}

\author{
Address: ${ }^{1}$ McGill University AIDS Centre, Montreal, Canada and ${ }^{2}$ Botswana-Harvard AIDS Partnership, Gaborone, Botswana \\ * Corresponding author
}

from 2006 International Meeting of The Institute of Human Virology

Baltimore, USA. 17-21 November, 2006

Published: 21 December 2006

Retrovirology 2006, 3(SuppI I):S4 doi:I0.I I86/I742-4690-3-SI-S4

(c) 2006 Wainberg et al; licensee BioMed Central Ltd.

\section{Background}

The K65R substitution can cause extensive cross-resistance among currently used NRTIs, yet this mutation has rarely been observed among subtype B infected individuals who receive antiretroviral (ARV) drugs.

\section{Methods}

We evaluated the incidence of K65R in subtype $C$ patients in Botswana, who received ARV therapy in the context of first and second line regimens by studying the reverse transcriptase (RT) genotypes of 23 individuals who failed therapy, while on combination regimens that included ddI and d4T.

\section{Results}

Ten of these individuals had initiated treatment with ddI/ $\mathrm{d} 4 \mathrm{~T}$-based regimens while 13 had started therapy with ZDV/3TC/NVP or ZDV/3TC/EFV prior to switching to ddI-and/or d4T-containing combinations. Of the 23 patients, 7 possessed K65R and no-one possessed L74V after a median exposure to combination $\mathrm{ddI} / \mathrm{d} 4 \mathrm{~T}$ of only eight months (range 4-18 months). In contrast, none of 13 patients who received 3TC/ZDV as initial therapy prior to $\mathrm{d} 4 \mathrm{~T} / \mathrm{ddI}$ developed K65R, although most of them developed thymidine-associated mutations (TAMs); this is potentially due to antagonism between TAMs and K65R. In tissue culture studies, K65R was detected after only 12 weeks in $4 / 4$ and 3/4 selections with Tenofovir and ddI, respectively, but far longer periods were required for relatively infrequent selection of K65R by Tenofovir with subtype B viruses (>40 weeks; 2/7 selections).

\section{Conclusion}

K65R may emerge at higher frequency in individuals infected with subtype $\mathrm{C}$ viruses who experience treatment with certain NRTIs, establishing the need to monitor for the presence of this mutation and its possible transmission. 